\title{
Entwicklungsfinanzierung: Eintrittsportale ins Internet
}

Lucas Oesch

\section{OpenEdition}

\section{Journals}

Electronic version

URL: http://journals.openedition.org/sjep/417

DOI: $10.4000 /$ sjep. 417

ISSN: 1663-9677

\section{Publisher}

Institut de hautes études internationales et du développement

\section{Printed version}

Date of publication: 1 novembre 2007

Number of pages: $261-270$

ISBN: 978-2-88247-069-0

ISSN: $1660-5926$

\section{Electronic reference}

Lucas Oesch, "Entwicklungsfinanzierung: Eintrittsportale ins Internet », Schweizerisches Jahrbuch für Entwicklungspolitik [Online], 26-2 | 2007, Online erschienen am: 06 September 2010, abgerufen am 08 September 2020. URL : http://journals.openedition.org/sjep/417 ; DOI : https://doi.org/10.4000/sjep. 417 


\title{
Entwicklungsfinanzierung: Eintrittsportale ins Internet
}

\author{
Lucas Oesch*
}

\section{Einführung}

Die vorliegende Hintergrundinformation umfasst eine Reihe ausgewählter Websites zum Thema „Entwicklungsfinanzierung“. Sie ist für Leserinnen und Leser gedacht, die ihre Kenntnisse zu dieser Thematik vertiefen wollen. Unter den zahlreichen Websites von internationalen Organisationen, Regierungen, Nichtregierungsorganisationen (NRO) und Forschungszentren, die sich mit Entwicklungsfragen beschäftigen, wurden solche ausgewählt, die einen Zusammenhang mit der Entwicklungsfinanzierung haben, ohne dass es sich dabei um eine vollständige Auswahl handelt.

Im 1. Teil werden Websites internationaler Organisationen präsentiert, die sich mit Entwicklungsfinanzierung befassen. Im 2. Teil werden die Websites einiger nationaler Regierungen in Europa und Nordamerika zum gleichen Thema aufgeführt. Im 3. Teil werden Websites von Nichtregierungsorganisationen und Forschungszentren zur Entwicklungsfinanzierung im Allgemeinen und danach zu speziellen Themen (Mikrofinanz, Überweisungen von MigrantInnen, Fiskalität, Korruption, Schulden und natürliche Ressourcen) vorgestellt.

\section{Internationale Organisationen}

\section{Financing for Development Office, United Nations}

\section{$<$ http://www.un.org/esa/ffd > (Englisch)}

Das Financing for Development Office wurde 2002 nach der internationalen Konferenz von Monterrey über Entwicklungsfinanzierung errichtet. Es soll die Umsetzung und Überprüfung der Abkommen und der an der Konferenz eingegangenen Verpflichtungen erleichtern. Die Website in englischer Sprache stellt Informationen über den Folgeprozess des „Konsenses von Monterrey“ bereit. Man findet dort hauptsächlich Resolutionen, Berichte und andere UN-Publikationen zur Entwicklungsfinanzierung in mehreren Sprachen. Die Website informiert auch über Massnahmen des zwischenstaatlichen Folgeprozesses (>Intergovernmental Follow-Up) sowie über diverse Konferenzen und Konsultationen, die bis zur zweiten Jahreshälfte 2008 vorgesehen sind, wenn die nächste Konferenz über Entwicklungsfinanzierung in Doha (Katar) stattfindet. In diesem Zusammenhang besteht die Möglichkeit, sich für einen monatlichen Newsletter

* Doktorand am Institut universitaire d'études du développement (IUED), Genf. 
mit dem Titel The Road to Doha einzutragen. Diese Website stellt eine unumgängliche Quelle mit Dokumenten aus erster Hand für alle dar, die sich für das Thema interessieren. Gleichzeitig bietet sie die Möglichkeit, sich über die Aktualität und die zwischenstaatlichen Massnahmen in diesem Bereich auf dem Laufenden zu halten.

Gender Responsive Budgeting, United Nations Development Fund for Women (UNIFEM)'

$<$ http://www.gender-budgets.org $>$ (Englisch, Französisch und Spanisch $)^{2}$

Dieses Internetportal wurde 2001 eingeführt. Es wird unter anderen vom Entwicklungsfonds der Vereinten Nationen für Frauen (UNIFEM) unterstützt und wurde 2007 erneuert. Ziel dieser Website ist es, Entwicklungsakteure für den Gender-Ansatz im Budgetprozess (Gender Responsive Budgeting) zu sensibilisieren. Ein Dokumentationszentrum (>Resource Center) bietet Zugang zu zahlreichen Unterlagen (Studien, Berichte, usw.) in Englisch, Französisch, Spanisch und Arabisch. Ferner ist eine nach Regionen geordnete Dokumentation unter (>Country Resources) zugänglich. Auf der Eingangsseite (>Home) findet man aktualisierte Meldungen des UNIFEM (>Message from UNIFEM).

\section{United Nations Conference on Trade and Development (UNCTAD)}

$<$ http://www.unctad.org> (Englisch, Französisch und Spanisch) Home >Programmes >International Investment Flows (World Investment Report)

Die UN-Konferenz über Handel und Entwicklung (UNCTAD) veröffentlicht alljährlich einen Bericht über Trends der ausländischen Direktinvestitionen (FDI) weltweit, mit Analysen der Auswirkungen auf die Entwicklung. Der Bericht mit dem Titel World Investment Report besteht nur in Englisch, jedoch sind Zusammenfassungen in mehreren Sprachen verfügbar.

\section{Development Assistance Committee (DAC), Organisation for Economic Co-operation and Development (OECD) \\ $<$ <ttp://www.oecd.org/dac> (Englisch und Französisch)}

Der Entwicklungshilfeausschuss (DAC) ist das Gremium der Organisation für wirtschaftliche Zusammenarbeit und Entwicklung (OECD), das sich mit Fragen der Entwicklungszusammenarbeit befasst. Mehrere Abteilungen der OECD beschäftigen sich mit dem Thema „Entwicklungsfinanzierung“. Nachstehend werden der Zugang und die Webseite, die als relevanteste zu diesem Thema angesehen wird, beschrieben. Die Website des DAC informiert im Wesentlichen über die allgemeinen Ausrichtungen der Entwicklungspolitik der Industriestaaten. Sie ist in englischer Sprache gehalten, jedoch besteht auch eine kürzere Version in Französisch.

\footnotetext{
Ich danke Fenneke Reysoo für seine Hinweise zu dieser Website.

Bei allen hier aufgeführten Websites ist die erstgenannte Sprache die Hauptsprache.
} 
Home $>$ Related Topics $>$ Development $>$ Finance for Development

Diese Website wird vom Entwicklungszentrum der OECD geführt, das eng mit dem DAC zusammenarbeitet. Sie stellt hautsächlich Studien, Arbeitsunterlagen, Orientierungs- und Synthesedokumente (Policy Insights und Policy Briefs) zu Themen der Entwicklungsfinanzierung bereit. Die Unterlagen können entweder unklassiert unter (>Publications \& Documents) oder nach Ländern geordnet unter (>Information by Country) abgerufen werden. Die Seite „Publications \& Documents“ enthält auch einen Link zu einer neuen, im Jahr 2007 eingeführten Jahrespublikation, den Bericht Financing Development: Aid and Beyond (>Annual Reports), der aber noch nicht komplett zugänglich ist. Ferner besteht ein Link zu einem Entwicklungsforum (>OECD Global Forum on Development), das sich im Zeitraum 2006-2009 mit dem Thema Financing Development Effectively beschäftigt. Die Berichterstattungen und Programme der verschiedenen Treffen im Rahmen des Forums sind dort erhältlich.

Home $>$ Related Topics $>$ Development $>$ Aid Effectiveness und/oder Investment for Development

Hierbei handelt es sich um zwei weitere Zugänge zu Themen der Entwicklungsfinanzierung.

\section{International Monetary Fund (IMF)}

$<$ http://www.imf.org > (Englisch, Französisch und Spanisch) Home >Publications $>$ Series Titles: Finance and Development $(F \& D)$

Die Vierteljahreszeitschrift Finance and Development wird vom Internationalen Währungsfonds in den Sprachen Englisch, Französisch und Spanisch herausgegeben. Die Zeitschrift befasst sich mit Finanz-, Entwicklungs- und Entwicklungsfinanzierungsfragen. Die Archive (seit 1996) und die neueste Nummer sind auf der Website zugänglich.

\section{World Bank}

$<$ http://www.worldbank.org > (Englisch, Französisch und Spanisch) Home $>$ Data \& Research $>$ Prospects $>$ Publications $>$ Global Development Finance

Der von der Weltbank publizierte Jahresbericht Global Development Finance ist der Entwicklungsfinanzierung in der Welt gewidmet und behandelt jedes Jahr ein damit zusammenhängendes neues Thema. Er erstellt insbesondere eine Diagnose der jüngsten Kapitalflüsse in die Entwicklungsländer und beschreibt die Zukunftsaussichten. Der Bericht in englischer Sprache ist seit der Ausgabe 1999 erhältlich. Ferner bestehen Zusammenfassungen in anderen Sprachen. 


\section{Europäische Union}

<http://europa.eu> (europäische Sprachen)

$\square$ EuropeAid Co-operation Office

$<$ http://ec.europa.eu/europeaid>

Diese Website bietet Zugang zu Mikrofinanztätigkeiten, die vom Europe Aid Co-operation Office unterstützt werden (>Programmes and Projects $>$ ACP/EU Microfinance). Die Dokumentationsseite ( $>$ Reports and Publications) enthält auch eine Reihe interessanter Unterlagen zu den verschiedenen Hilfezuteilungsmethoden (Aid Delivery Methods): Projekthilfe, Budgethilfe, usw.

\section{Schweiz}

$<$ http://www.admin.ch> (Englisch, Französisch, Deutsch und Italienisch)

Zwei Ämter der Bundesverwaltung befassen sich besonders mit Entwicklungsfinanzierungsfragen:

$\square$ Direktion für Entwicklung und Zusammenarbeit (DEZA)

$<$ http://www.deza.admin.ch>Home >Themen >Wirtschaft und Beschäftigung $>$ Finanzsektorentwicklung

Diese Webseite stellt die Arbeit der Direktion für Entwicklung und Zusammenarbeit (DEZA) zur Förderung des Zugangs der Bevölkerung in den Entwicklungsländern zu Finanzdienstleistungen (Sparwesen, Kredit, Geldüberweisungen und Versicherungen) vor. Ferner ist dort eine Dokumentation betreffend die verschiedenen Finanzdienstleistungen zu finden.

$\square$ Leistungszentrum Wirtschaftliche Entwicklung und Zusammenarbeit

(Staatssekretariat für Wirtschaft, SECO)

$<$ http://www.seco-cooperation.admin.ch> Home > Themen >Makroökonomische Unterstützung

Diese Seite beschreibt die Tätigkeit des Staatssekretariats für Wirtschaft (SECO) im Bereich der Budgethilfen und der Hilfe zur Errichtung von Finanzund Bankensektoren in den Entwicklungsländern. Zudem werden die vom Bund unterstützten Entschuldungsmassnahmen präsentiert.

\section{Frankreich}

$\square$ Ministère des affaires étrangères et européennes $<$ http://www.diplomatie.gouv.fr> (Französisch, Englisch, Deutsch und Spanisch) Accueil >Actions de la France > Financements innovants du développement 


\section{Deutschland}

$\square$ Deutsche Gesellschaft für Technische Zusammenarbeit (GTZ)

$<$ http://www.gtz.de>(Deutsch und Englisch)

Startseite $>$ GTZ - Themen $>$ Wirtschaft und Beschäftigung $>$ Finanzsysteme

Startseite $>$ GTZ - Themen $>$ Good Governance $>$ Öffentliche Finanzen und/oder Korruption

\section{Grossbritannien}

$\square$ Department for International Development (DFID)

$<$ http://www.dfid.gov.uk> (Englisch), Home > Millennium Dev't Goals > Aid,

Trade, Growth \& Global Partnership >Aid effectiveness

\section{Vereinigte Staaten}

$\square$ United States Agency for International Development (USAID)

$<$ http://www.usaid.gov> (Englisch) Home >Our Work > Economic Growth \& Trade

\section{Kanada}

$\square$ Canadian International Development Agency (CIDA)

$<$ http://www.acdi-cida.gc.ca> (Englisch und Französisch) Home >Publications $>$ Policy Suite > Policy Statement on Strengthening Aid Effectiveness

\section{Nichtregierungsorganisationen (NRO) und Forschungszentren}

\section{Eldis}

$<$ http://www.eldis.org > (Englisch)

Hierbei handelt es sich um ein Internetportal, das vom Institute of Development Studies (IDS) verwaltet und von verschiedenen nationalen Entwicklungsagenturen finanziert wird. Unter der Rubrik (>Search Eldis) können Dokumente von in der Entwicklung tätigen Organisationen gesucht werden. Zur Suche lassen sich die Stichwörter „financing development" verwenden.

\section{Europe's Forum on International Cooperation (EUFORIC)}

<http://www.euforic.org>(Englisch und Französisch)

Dieses europäische Forum über internationale Zusammenarbeit (EUFORIC) wird von diversen nationalen Entwicklungsagenturen, Nichtregierungsorganisationen und Entwicklungsforschungsinstituten unterstützt. Auf der Suchseite (>Search) hat man mittels vier verschiedener Suchmaschinen Zugang zu 
Dokumenten und zu anderen Websites in Verbindung mit der Entwicklungsthematik. Die Stichwörter „financing development“ ergeben interessante Resultate in den vier Suchmaschinen.

\section{World Institute for Development Economics Research, United Nations University (UNU-WIDER) \\ $<h t t p: / / w w w . w i d e r . u n u . e d u>$ (Englisch)}

Die Suchrubrik dieser Website (>Search) bietet Zugang zu Forschungsarbeiten und Diskussionspapieren in englischer Sprache, die von den Forschern des World Institute for Development Economics Research (WIDER) der Universität der Vereinten Nationen erstellt wurden. Die Stichwörter „,finance“ oder „financing" führen zu interessanten Unterlagen über Entwicklungsfinanzierung hin.

\section{Deutsches Institut für Entwicklungspolitik (DIE)}

$<$ http://www.die-gdi.de> (Deutsch, Englisch, Französisch und Spanisch)

Home >Forschung und Beratung >Forschungsfeld IV: Abteilung V: Weltwirtschaft und Entwicklungsfinanzierung

Home $>$ Research and Consultancy $>$ Focal area IV: Dept. V: World Economy and Development Financing

Auf der Website des Instituts (DIE) werden die Abteilung V und ihre verschiedenen Forschungsprojekte zu Wirtschafts- und Entwicklungsfinanzierungsfragen vorgestellt.

\section{World Economic Forum (WEF)}

$<$ http://www.weforum.org $>$ (Englisch) Home $>$ Initiatives

$>$ Financing for Development

Die Website des Weltwirtschaftsforums stellt die öffentlich-privaten Partnerschaften (PPP) und die privaten Investitionen als potenzielle Entwicklungsinstrumente heraus.

\section{Gerster Consulting (Gerster Development Consultants)}

$<$ http://www.gersterconsulting.ch $>$ (Englisch) Home $>$ Results $>$ Finance

Die Website Gerster Consulting bietet Zugang zu den Arbeiten des Beratungsbüros bezüglich Finanzierungsfragen. Man findet dort ein im Jahr 2005 für die Direktion für Entwicklung und Zusammenarbeit (DEZA) erstelltes Dokument mit dem Titel New Sources of Development Financing : An SDC Working Paper. 


\section{Global Policy Forum (GPF)}

$<$ http://www.globalpolicy.org > (Enlisch) Home >Social \& Economic Policy $>$ Financing for Development

Die Nichtregierungsorganisation Global Policy Forum (GPF) hat sich zum Ziel gesetzt, die Beschlüsse und Politiken der Vereinten Nationen, namentlich im Entwicklungsfinanzierungsbereich zu verfolgen und zu analysieren.

\section{Choike (Third World Institute, ITEM)}

$<$ http://www.choike.org > (Englisch und Spanisch) Home >In-depth Reports $>$ Society: Financing for Development

Hierbei handelt es sich um ein Internetportal, das von der NRO Third World Institute (ITEM) verwaltet wird. Die Seite über Entwicklungsfinanzierung führt insbesondere eine Reihe interessanter Links (Related links) auf.

\section{Coordination Sud}

$<$ http://www.coordinationsud.org > (Französisch) Accueil

$>$ Positions des $O N G>$ Financement $d u d v l p t$

Dieses Webportal französischer Nichtregierungsorganisationen, die sich im Bereich internationaler Solidarität einsetzen, informiert namentlich über aktuelle Fragen der Entwicklungsfinanzierung.

\section{European NGO Confederation for Relief and Development (CONCORD)}

$<$ http://www.concordeurope.org > (Englisch und Französisch) Home > Topics $>$ Financing for development - Aidwatch

Dieses Netzwerk europäischer NRO (CONCORD) bietet insbesondere eine Übersicht und Analyse über Umfang und Qualität der öffentlichen Entwicklungshilfe, die von der Europäischen Union und den Mitgliedsstaaten aufgebracht wird.

\section{- Mikrofinanz}

\section{Mikrofinanzportal}

<http://www.lamicrofinance.org > (Französisch und Englisch)

Dieses Internetportal wird von verschiedenen nationalen Entwicklungsagenturen, Nichtregierungsorganisationen und Forschungsinstituten unterstützt. Es bietet eine grosse Anzahl Unterlagen zu Themen der Mikrofinanz (Themendossiers) (>Dossiers thématiques) an. Auch die Suche mit Stichwörtern ist unter der Rubrik (>Bibliothèque) möglich. Eine Rubrik mit aktualisierten Kurznachrichten (>Actualités) erlaubt zudem, sich über die Entwicklung im Mikrofinanzbereich auf dem Laufenden zu halten. Schliesslich enthält eine Linkseite (Autres ressources >Liens) eine detaillierte Beschreibung weiterer Websites zum Thema Mikrofinanz. 


\section{Forum finances et développement (Epargne sans frontière, ESF)}

$<$ http://esf.asso.fr> (Französisch und Englisch

Dieses Webportal wird von der Vereinigung Epargne sans frontière (ESF) unterhalten. Wie das vorhergehende Portal ermöglicht es, sich von der Eingangsseite (>Accueil du site) ausgehend über verschiedene Themen der Mikrofinanz zu informieren. Auch bietet es Zugang zu der von der Vereinigung publizierten Zeitschrift Techniques financières et développement (>La revue: Techniques financières et développement). Des Weiteren besteht die Möglichkeit der Beteiligung an Diskussionsforen unter der Rubrik (>Nos forums).

\section{- Überweisungen von Migrantlnnen}

\section{Remittances.eu}

(Foundation for International Migration and Development, FIMD)

<http://www.remittances.eu> (Englisch)

Auf der Eingangsseite (>Home) bietet die Website der Stiftung (FIMD) Zugang zu Kurznachrichten und zu den neuesten Publikationen zum Thema „Überweisungen von Migrantinnen und Migranten“ (Newest publications). Ferner sind Exemplare eines Newsletter mit dem Titel Migrant Remittances einzusehen.

\section{- Fiskalität}

\section{Tax Justice Network}

<http://www.taxjustice.net/cms> (Englisch, Französisch, Deutsch und Spanisch) Auf der Website der NRO Tax Justice Network werden Erläuterungen zu verschiedenen Themen im Zusammenhang mit der Fiskalität (Steuerbetrug, Steuerwettbewerb, usw.) unter der Rubrik (>Resources) präsentiert. Zum Zeitpunkt der Abfassung dieser Hintergrundinformation waren allerdings noch viele Rubriken im Aufbau begriffen. Unter der Nachrichtenrubrik (News) sind aktualisierte Links zu Zeitschriftenartikeln und Pressemitteilungen sowie Berichte und Konferenzanzeigen verfügbar.

\section{Interportal.ch}

$<$ http://www.interportal.ch> (Englisch, Französisch und Deutsch) Home >Contenu $>$ Dossiers $>$ Evasion fiscale, concurrence fiscale et pays en développement

Dieses Internetportal wird von Schweizer Nichtregierungsorganisationen finanziert. Es informiert über zahlreiche Themen bezüglich Entwicklung und internationale Entwicklungszusammenarbeit, namentlich über Steuerfragen im Rahmen der Entwicklungsfinanzierung. 


\section{- Korruption}

\section{Transparency International}

$<$ http://www.transparency.org $>$ (Englisch)

Diese Website führt die Arbeitsthemen der NRO Transparency International unter (>Global priorities) auf. Sie stellt eine Auswahl der Publikationen (>Publications) sowie Leitfäden bezüglich Analyse und Korruptionsbekämpfung (>Policy and research) bereit. Unter der Rubrik (>News room) besteht eine aktualisierte Webseite mit Pressemitteilungen der Organisation und ihrer diversen Regionalstellen.

\section{- Schulden}

\section{European Network on Debt and Development (EURODAD)}

$<$ http://www.eurodad.org > (Englisch, Französisch und Spanisch)

Die Website dieses Netzwerks europäischer NRO (EURODAD) bietet über zwei verschiedene Rubriken (Debt/Aid) eine Beschreibung von Themen zur Entwicklungshilfe und zur Schuldenfrage (diverse Arten von Schulden, Wirksamkeit der Hilfe, usw.). Zu jedem Thema sind Berichte und aktuelle Artikel verfügbar.

\section{Comité pour l'annulation de la dette du tiers-monde (CADTM)}

$<$ http://www.cadtm.org > (Englisch, Französisch und Spanisch)

Die Website des Komitees für die Streichung der Schulden der Dritten Welt (CADTM) stellt ebenfalls zahlreiche Informationen über Schuldenfragen (Zahlen, aktuelle Nachrichten, usw.) bereit.

\section{- Natürliche Ressourcen}

\section{The Extractive Industries Transparency Initiative (EITI)}

$<$ http://www.eitransparency.org $>$ (Englisch)

Die Website der Initiative für Transparenz in der Rohstoffwirtschaft (EITI) wird zum Teil von der Weltbank und von verschiedenen Regierungen finanziert. Sie bietet eine Übersicht über die Gouvernanz der natürlichen Ressourcen (Erdöl, Gas, usw.) in den rohstoffreichen Ländern (>EITI Countries). Unter der Rubrik (>Publications) werden eine Reihe von Berichten und Büchern zu dieser Thematik aufgeführt. Ferner enthält die Website eine aktualisierte Seite mit Presse$\operatorname{artikeln}(>$ EITI News). 


\section{Publish What You Pay coalition}

$<$ http://www.publishwhatyoupay.org> (Englisch, Französisch, Deutsch, Spanisch und andere Sprachen)

Die Website dieser NRO-Koalition, die sich für mehr Transparenz bezüglich der Zahlungen der Bergbaugesellschaften und der Einkommen der Regierungen einsetzt, bildet ebenfalls eine Informationsquelle zu diesem Thema. 